\title{
Numerical simulation of a coarsening two-dimensional network
}

\author{
C. W. J. Beenakker \\ Philips Research Laboratories, NL-5600 JA Eindhoven, The Netherlands
}

(Received 14 October 1987)

\begin{abstract}
Topological correlations in a coarsening two-dimensional soap froth or polycrystalline network are studied by computer simulation. With use of a continuum model, grain growth in very large systems of over $10^{5}$ grains can be simulated. The correlations found between the size or the number of vertices of adjacent grains are in accordance with semiempirical rules of metallurgy. The average grain size grows in proportion to the square root of time, as predicted by mean-field theory. This result, that correlation effects do not modify the growth exponent, is consistent with dynamical scaling and agrees with simulations done on a lattice.
\end{abstract}

\section{INTRODUCTION}

Grain boundaries in a polycrystalline material are an interesting example of a system with topological disorder. ${ }^{1}$ Their motion is governed by the interplay of a "geometrical" driving force (which tries to shorten the boundaries) and the topological constraint of a spacefilling network. As a result of this interplay, correlations develop between the geometrical and topological properties of the grains. These correlations are easy to visualize in a two-dimensional (2D) model of the grain growth process. For this model, various semi-empirical rules to describe the correlations have been proposed, ${ }^{2-4}$ obtained from cross sections of polycrystalline metals or ceramics, and from an analogous system: a soap froth sandwiched between two plates.

Even for the simple 2D model, analytical progress beyond mean-field theory ${ }^{5-9}$ has not been made. For this reason numerical studies play an important role in the theory of grain growth. In recent years, the Potts lattice model has been studied extensively. ${ }^{10,11}$ In the present paper, an alternative continuum model is investigated, which is based on (i) the macroscopic growth law ${ }^{12,13}$ of a grain, and (ii) a clever rule due to Marder ${ }^{14}$ for deciding when a grain loses or gains a vertex. As we will see, this approach allows the study of very large systems (consisting initially of over $10^{5}$ grains) which can be followed for several decades in time-before the number of grains has dropped to the point where effects of the finite system size become noticeable. Long coarsening times are important for a full development of the dynamical correlations, which asymptotically become independent of initial conditions.

In Sec. II the method used is presented, and its relation to both 2D grain growth and coarsening soap froths is explained. The results are discussed in Sec. III. In the asymptotic regime a negative correlation is found between the number of vertices as well as the size of adjacent grains. The vertex-number correlation agrees with the Aboav-Weaire rule ${ }^{2,3}$ of metallurgy, which says that many-sided grains have few-sided neighbors. The size correlation is similar to that found in a simulation ${ }^{15}$ of the coarsening of precipitated droplets (Ostwald ripen- ing). It is well established ${ }^{16,17}$ that in Ostwald ripening, correlation effects do not modify the mean-field value of the exponent $\alpha$ in the equation

$$
\bar{R}(t)=\text { const } \times t^{\alpha},
$$

which describes the time dependence of the average droplet radius. In both $2 \mathrm{D}$ and $3 \mathrm{D}$, one has $\alpha=\frac{1}{3}$ for diffusion-limited droplet growth and $\alpha=\frac{1}{2}$ if the diffusion of a solute molecule from one droplet to another is much faster than its attachment at the droplet surface. Clearly, grain growth is governed by the boundary kinetics, so that one would expect to find $\alpha=\frac{1}{2}$ regardless of correlation effects - at least if one assumes that the presence of topological constraints is not a fundamental distinction between the coarsening of cells in a network and of separated droplets. This has been a controversial issue, ${ }^{10,11,14,18-22}$ which is addressed in Sec. III in relation to the simulation.

\section{METHOD}

The starting point of this investigation is Mullins's area theorem $^{12}$

$$
\frac{d A}{d t}=\frac{\pi}{3} M(n-6),
$$

which relates the time derivative of the area $A$ of a $2 \mathrm{D}$ grain to its number of sides $n$ and the grain boundary mobility $M$. Equation (2) follows via simple geometry from (i) the curvature law ${ }^{23}$ that each segment of a grain boundary moves towards its center of curvature with a velocity $V$ proportional to the curvature $\Gamma$,

$$
V=M \Gamma,
$$

and (ii) the local equilibrium condition at the vertices that in the infinitesimal region of intersection three grain boundaries meet at equal angles of $120^{\circ}$. Note that Eq. (2) implies that the total area of the system remains constant in time, as it should, by virtue of Euler's theorem that the grains have six sides on average. In the case of a soap froth, the curvature law is replaced by Laplace's law, together with a linear relation between pressure 
differences in adjacent cells and the gas flux through the cell walls. The result in 2D is still Eq. (2), with $M$ replaced by the product of soap film permeability $\mu$ and surface tension coefficient $\sigma$. Equation (2) is known in that context as von Neumann's theorem. ${ }^{13}$ Von Neumann's derivation assumes circular soap-cell boundaries, as required by pressure equilibration inside the soap cells. It should be stressed that Mullins's derivation of Eq. (2) does not assume that grain boundaries are circular arcs, which indeed they are not.

The fact that the shape of the grain boundary does not appear in the growth law (2) is characteristic for two dimensions. In 2D the curvature of a grain boundary equals $d \phi / d l$ ( $\phi$ being the angle of the tangent to the boundary and $l$ its length). The integral of the curvature along the perimeter of a grain is, therefore, simply

$$
\oint d l \Gamma=\oint d l \frac{d \phi}{d l}=2 \pi-\sum_{i=1}^{n}\left(\pi-\alpha_{i}\right)=-\frac{\pi}{3}(n-6),
$$

regardless of the shape of the boundary. Here $\alpha_{1} \cdots \alpha_{n}$ are the internal angles at the $n$ vertices of the grain, which equal $2 \pi / 3$ at local equilibrium. The time derivative of the area is proportional to the above integral,

$$
\frac{d A}{d t}=- \text { const } \times \oint d l \Gamma,
$$

where the constant equals $M$ for grain boundaries and $\mu \sigma$ for soap films. The growth law (2) now follows from Eqs. (4) and (5).

The power of Eq. (2) is that it allows a reduced description of a coarsening network in terms of only two variables $A$ and $n$ per grain, when supplemented by a model ${ }^{14}$ for changes in $n$. By contrast, other simulations either ${ }^{24}$ achieve such reduction by doing away with the network structure (treating the grains as separated spherical "droplets"), or ${ }^{10,11,25,26}$ employ a full description in which the motion of every boundary segment is followed in time. Note that the reduction in the number of variables works only in 2D. Unlike the Potts model, ${ }^{27}$ the present model has no obvious extension to three dimensions.

To account for the dynamics of $n, I$ adopt a simple rule due to Marder. ${ }^{14}$ In principle, a grain can change its number of sides either by a neighbor-switching " $\mathrm{T} 1$ process," or by a "T2 process" which involves the disappearance of a grain, see Fig. 1. In the experiments of Glazier, Gross, and Stavans, ${ }^{21}$ it is observed that in a coarsening soap froth only, a small fraction of the topological changes occur via neighbor switching without a disappearing cell. It seems reasonable to assume that the $\mathrm{T} 1$ processes are also relatively unimportant during grain growth, and the present model neglects them.

My algorithm now goes as follows. For each grain $i=1,2, \ldots, N$ track is kept of its area $A_{i}$, its number of sides $n_{l}$, and its neighbors $j_{l, k}\left(k=1,2, \ldots, n_{l}\right.$, listed clockwise). The areas are updated according to Eq. (2), and whenever the area of a grain drops to zero, that grain is eliminated via a $T 2$ process. When a four- or five-sided grain disappears, one would need to know which sides
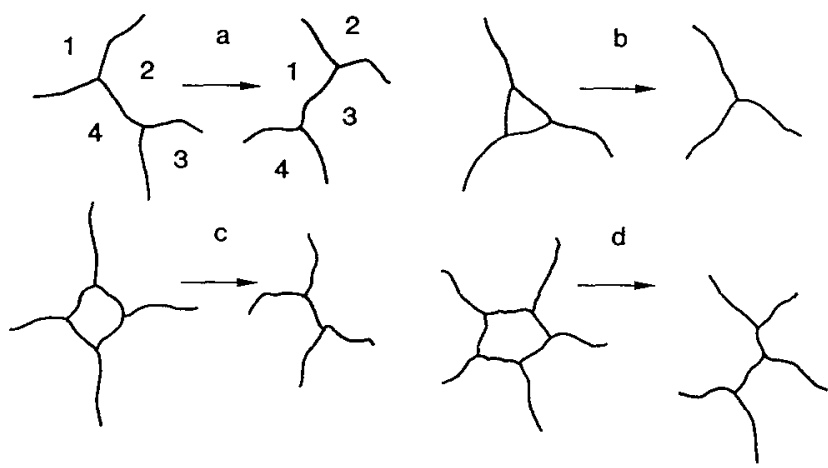

FIG. 1. Sketch of grain boundaries undergoing topological changes. (a) shows the $\mathrm{T} 1$ or neighbor-switching process; (b)-(d) show T2 processes related to a disappearing 3-, 4-, or 5 sided grain. [Notice how in (d) one grain ends up gaining a side.]

vanish first to make the choice which of its neighbors end up losing a vertex in the $\mathrm{T} 2$ process. However, since grains tend to be equi-axed, one may well assume that all sides are equivalent and make this choice at randomwhich is what I have done. The above scheme is rather tedious to code because of the bookkeeping required. (If one would not keep track of the network topology but ignore correlations between different grains it would essentially reduce to Marder's mean-field algorithm. ${ }^{14}$ ) What is important is that the program is executed very rapidly, a run with initially $10^{5}$ grains taking only $10 \mathrm{~min}$ of central processing-unit time on an IBM-3081 computer.

Initially, the system studied consists of 102400 grains in either an ordered or disordered $2 \mathrm{D}$ array with periodic

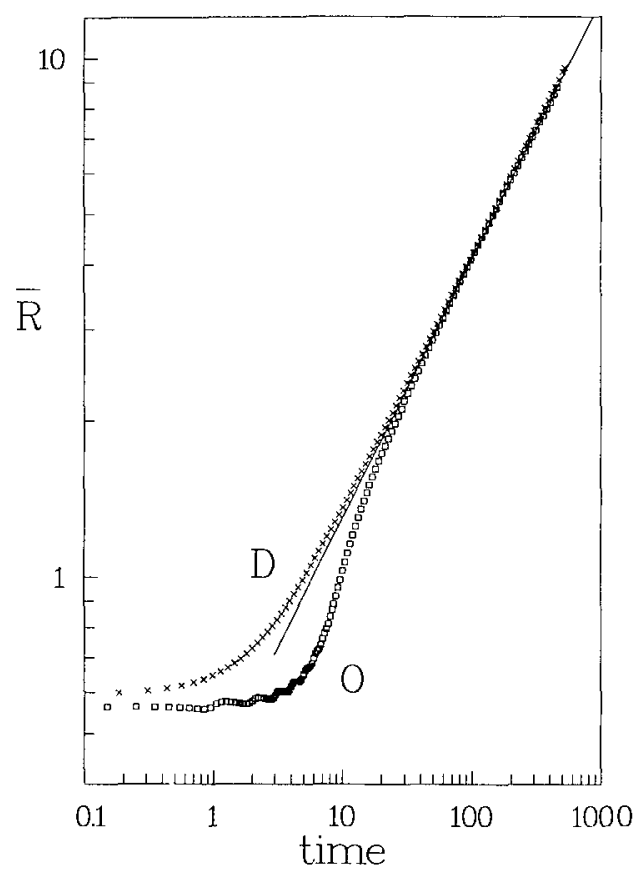

FIG. 2. Time dependence of the average grain size, for ordered $(O)$ and disordered $(D)$ initial conditions. Data points are averages over 10 runs. The solid line has a slope of $\frac{1}{2}$. (Time in units of $3 \bar{A}(0) / \pi M$, and $\bar{R}$ in units of $[\bar{A}(0) / \pi]^{1 / 2}$, with $\bar{A}(0)$ the initial average grain area.) 
boundary conditions. ${ }^{28}$ In both cases the same state of dynamical scaling is reached, although the way of approach to the asymptotic regime differs. In the scaling regime the distribution of the number of sides of a grain is time independent, and the distribution of grain sizes $P(R, t)$ is time invariant when expressed as a function of $R / \bar{R}(t)$. [The grain "size" $R$, with average $\bar{R}$, is defined by $R \equiv(A / \pi)^{1 / 2}$.] The asymptotic time dependence of $\bar{R}(t)$ obeys Eq. (1) with $\alpha=0.50$, see Fig. 2. Dynamical correlations are observed between the number of vertices of adjacent grains, as well as their sizes. These correlations are illustrated in Figs. 3 and 4, where I have plotted the time dependence of the correlation coefficients

$$
\begin{aligned}
& \chi \equiv N^{-1} \sum_{i=1}^{N}\left(n_{i} / 6-1\right)\left(v_{i} / 6-1\right), \\
& \psi \equiv N^{-1} \sum_{i=1}^{N}\left(R_{i} / \bar{R}-1\right)\left(\rho_{i} / \bar{R}-1\right) .
\end{aligned}
$$

The symbols $\rho_{i}$ and $v_{i}$ denote, respectively, the average size and average number of vertices of the grains adjoining $^{29}$ grain $i$ (which itself has size $R_{i}$ and $n_{i}$ vertices): The negative values of $\chi$ seen in Fig. 3 imply that manysided grains tend to be surrounded by few-sided neighbors, and, similarly, a negative $\psi$ means that large grains have small neighbors.

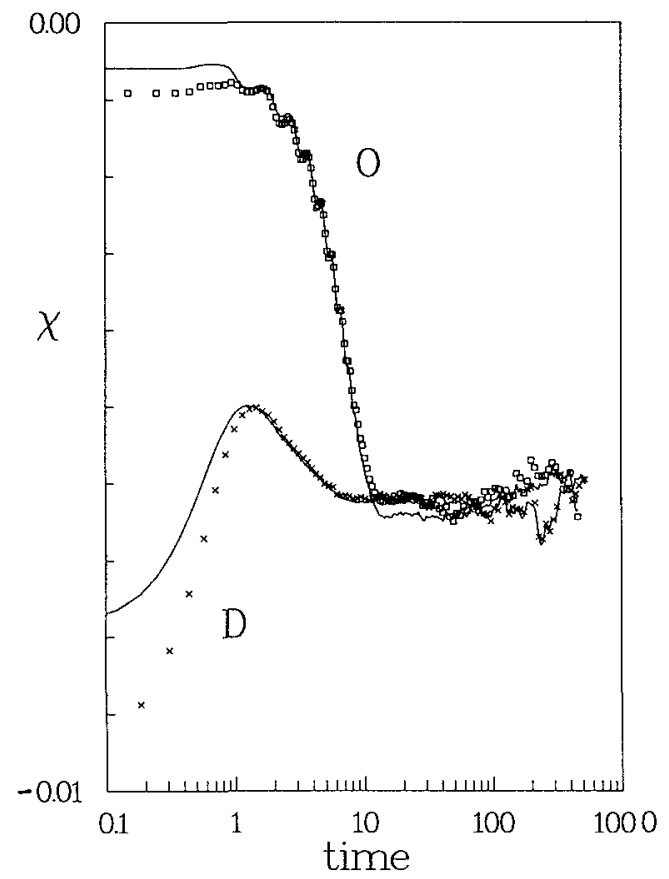

FIG. 3. Time dependence of the vertex-number correlation coefficient $\chi$, defined in Eq. (6a). Data points $O$ and $D$ as in Fig. 2. The solid lines are predicted by the Aboav-Weaire rule. Note the transient "dips" in curve $O$, characteristic for the initially ordered system, which signal the sudden disappearance of a substantial fraction of the grains. (The transient "steps" in Fig. 2, case $O$, have the same origin.) The noise in the data increases somewhat with time, because of the decreasing number of grains - which drops from $10^{5}$ to 300 during one run.

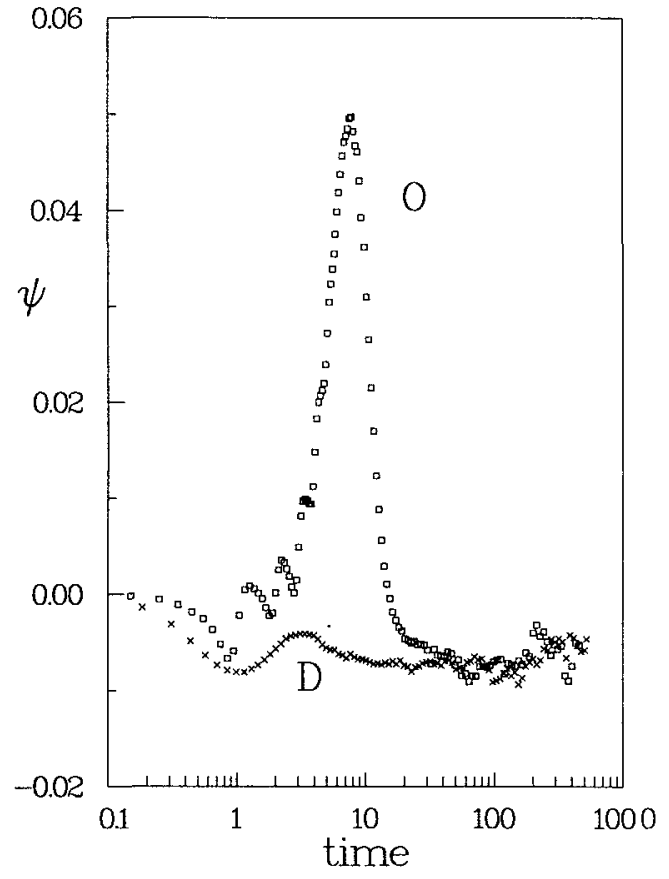

FIG. 4. Time dependence of the size-correlation coefficient $\psi$, defined in Eq. (6b). Data points $O$ and $D$ as in Fig. 2.

\section{DISCUSSION}

Before proceeding to a discussion of the correlations, I will first compare the results for the single-grain distribution functions obtained here with those from the Potts lattice model simulations.

The time-invariant distribution of grain sizes $P(R / \bar{R})$ compares well with the distribution of Sahni et al., ${ }^{10}$ see Fig. 5. Although obtained from 2D simulations, Fig. 5 happens to give a good description of cross sections of bulk metals and ceramics, see Refs. 10 and 27. Apparently no experimental data for $P(R / \bar{R})$ in sheet material are

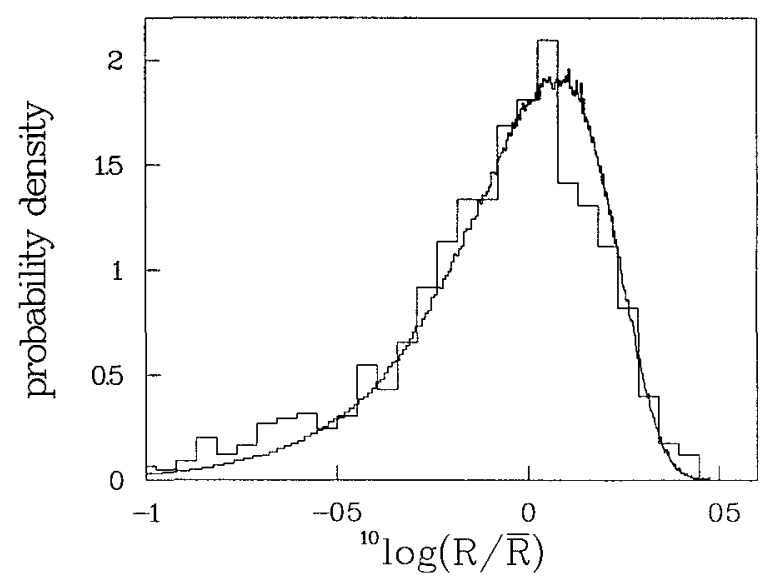

FIG. 5. Distribution of grain sizes in the long-time regime. The histogram with the higher resolution follows from the present model, the other from the Potts model of Ref. 10. As is customary, the distribution is given as a function of $x \equiv{ }^{10} \log (R / \bar{R})$. The probability density $P(x)$ is such that $P(x) d x$ equals the fraction of grains with $x^{\prime}$ between $x$ and $x+d x$. 
available. For the distribution of the number of sides $P(n)$, Simpson, Beingessner, and Winegard ${ }^{30}$ have collected measurements on a variety of quasi-2D systems (two polycrystalline materials and soap). Their data are shown in Fig. 6 together with the theoretical predictions of this paper and of Sahni et al. It seems likely that differences in the frequency of neighbor-switching processes are responsible for the variations in $P(n)$ between the two models. In fact, these $\mathrm{T} 1$ processes tend to broaden $P(n)$, consistent with the observation that the model used here (in which $\mathrm{T} 1$ processes do not occur at all) gives a more narrow distribution than the model of Sahni et al. The experimental distributions have a width intermediate between the two predictions, and do not appear to favor one model above the other. A third singlegrain property of interest is the shape-size correlation expressed by the perimeter rule

$$
\bar{R}(n)=\frac{1}{4}(n-2) \bar{R},
$$

$\bar{R}(n)$ being the average size of an $n$-sided grain. In my opinion $^{31}$ this correlation should be seen as a consequence of the shape-dependent growth rate (2) of the grains: a large grain is likely to have many sides because many sided grains grow rapidly. A different point of view, based on a maximum entropy postulate, is taken by Rivier. ${ }^{4}$ The present model gives $\bar{R}(n)$ in good agreement with experiment, see Ref. 31 for a detailed comparison.

We now turn from the single-grain properties to pair correlations. The vertex-number correlation coefficient $\chi$ shown in Fig. 3 is in accordance with a semi-empirical rule of metallurgy, the so-called Aboav-Weaire rule, which says that

$$
\bar{v}(n)=5+(6+\mu) / n \text {. }
$$

Here $\mu \equiv\left\langle(n-6)^{2}\right\rangle$ is the variance of the number of sides distribution, and $\bar{v}(n)$ is the average of $v_{i}$ taken over all $n$-sided grains $i$. This rule says that a many-sided grain is likely to be surrounded by few-sided neighbors,

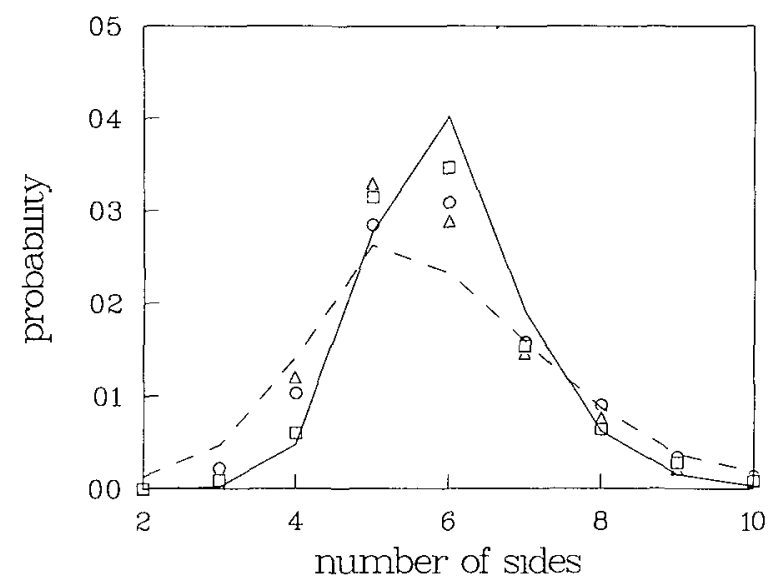

FIG. 6. Plot of the fraction of grains with a given number of sides in the long-time regime. Solid curve from the present model, dashed curve from the Potts model of Ref. 10. The experimental data are from Ref. 30 for quasi-2D systems (triangles, polycrystalline tin; squares, polycrystalline hexachloroethane; circles, soap froth). as has been observed in $2 \mathrm{D}$ soap-froth experiments ${ }^{32}$ and simulations, ${ }^{26}$ as well as in sectioned polycrystalline materials. $^{2}$ A statistical argument for such correlation is given by Weaire. ${ }^{3}$ (A more complicated variant of this rule is also in use, see Refs. 32 and 33.) From the Aboav-Weaire rule follows the prediction $\chi=-(1$ $+\mu / 6)(\langle 1 / n\rangle-1 / 6)$, which describes very well the data in Fig. 3-except during an initial transient. The size correlation of Fig. 4 has not yet been studied experimentally. The perimeter rule suggests the estimate

$$
\psi \approx\left\langle\left[\frac{1}{4}(n-2)-1\right]\left[\frac{1}{4}(\nu-2)-1\right]\right\rangle=\frac{9}{4} \chi .
$$

This rough estimate predicts the sign and within a factor of 2 the magnitude of $\psi$ in the scaling regime, but cannot explain the remarkable positive correlation peak observed in the initially ordered system.

It is interesting to note that the asymptotic size correlation between these neighboring polycrystalline domains is of the same sign (but an order of magnitude smaller ${ }^{34}$ ) as that found in a simulation ${ }^{15}$ of Ostwald ripening. (Ostwald ripening is the growth of precipitated droplets by diffusion of solute through a liquid or solid solution.) For the latter problem perturbation theories ${ }^{16,17}$ have been developed, with the volume fraction of the dispersed precipitate as the small parameter. Such an expansion parameter is not available in the network, but the physical origin of the size correlation is presumably the same as in Ostwald ripening: large grains have grown at the expense of their neighbors, which will therefore be small.

Now that we have seen that the present model can reproduce the correlations in grain growth, let us consider the fundamental issue: Do topological correlations modify the kinetic exponent $\alpha$ in Eq. (1)? Figure 2 shows that the mean-field value $\alpha=\frac{1}{2}$ remains unmodified. This conclusion is consistent with the hypothesis of dynamical scaling, which says that the average grain size $\bar{R}$ is the only independent length scale in the system. Indeed, it is a consequence of this hypothesis that the length $\lambda \equiv \sqrt{t M}$ is identical to $\bar{R}$ up to a multiplicative numerical constant-which implies ${ }^{35} \mathrm{Eq}$. (1) with $\alpha=\frac{1}{2}$. [Here $M$ is the grain boundary mobility, defined via the curvature law (3); in soap froths, $\lambda \equiv \sqrt{t \mu \sigma}$ with $\mu$ the soap-film permeability and $\sigma$ its surface tension coefficient.] Clearly, the scaling hypothesis requires both the long-time limit and a large system, so that the lengths which characterize the initial condition and the system size do not play a role. In the case of a lattice model there is the additional requirement that the average grain size should be much larger than the lattice constant - to avoid the introduction of an additional relevant length scale. If this requirement is not met, the concepts of grain boundary curvature and mobility become meaningless, and the above argument fails.

In light of the above we can try to understand why previous workers ${ }^{10,11}$ obtained an anomalous $\alpha=0.41 \pm 0.02$ when simulating grain growth on a lattice-whereas a lattice model of soap-froth coarsening gave ${ }^{11} \quad \alpha=\frac{1}{2}$, in agreement with Fig. 2. I estimate from the information given in Ref. 10 that $\bar{R} \leqslant 10$ lattice constants. This does not seem especially large and deviations from the curvature law of motion (3) due to kinks in the boundary might 
well be the origin of the anomalous $\alpha$ After the present work was completed, I learned that Grest, Anderson, and Srolovit ${ }^{36}$ have extended their Potts model to larger lattices They indeed find a crossover to $\alpha=\frac{1}{2}$ at late times, when the grain boundaries become sufficiently smooth that one would expect the curvature law to describe the dynamics On the other hand, the soap-tioth simulation of Wejcheit, Wearre, and Kermode, ${ }^{11}$ as well as the present work, are directly based on the macroscopic growth law (2) Therefore, the network has a well-defined mobility $M$ (or $\mu \sigma$ product) at all times-and the simulations give $\alpha=\frac{1}{2}$ in agi eement with the scaling hypothesis

The experimental evidence semains inconclusive ${ }^{37}$ In polycrystalline matelials, $\bar{R} \propto \sqrt{t}$ is found in some systems, ${ }^{30}$ but one observes a general tendency for slower growth of the grains In metal films this can be accounted for by the pinning of grain boundanes at surface grooves In bulk samples, deviations from $\alpha=1$ have been attributed to the obstruction of grain boundary migration by impurity atoms These are additional complications, which may be removed as pure and purer systems become avallable

In soap froths, much less expenimental work has been done Early expeiıments by Smith (analyzed in Refs 18 and 32) showed no sign of a scaling regime, but instead gave a distribution $P(n)$ winch broadened steadily without reaching a time-1ndependent limiting form In Ref 20 I have proposed an explanation for this anoma- lous nonscaling behavior, based on the assumption that the many-sided shape of large soap cells is due to the relatively low sufface energy of such nearly circular cells The new expeı1ments by Glazıer, Gioss, and Stavans, ${ }^{21}$ however, disagree with the predictions of Ref 20 - and I now believe that this surface-energy mechanism plays only a minor tole in the dynamics The point is, as argued by Marder, ${ }^{14}$ that the soap film network is highly constrained in its movements and cannot easily reach an energetically more faroiable stıucture Marder's calculations show convincingly that the general features of these new experiments can be explained without considering the surface-energy mechanism [and also suggest that the anomalous broadening of $P(n)$ seen earlies was a transient effect] Glazier, Gross, and Stavans observe slowe1 than $\sqrt{t}$ growth of the soap-cell size The origin of this anomaly is unclear, both finite-size effects and variations in $\mu$ and $\sigma$ with film thickness may play a role

In summary, a method for simulating coarsening of very large $2 \mathrm{D}$ networks is reported, which reveals inter esting correlations and which demonstrates the valıd1ty of dynamical scaling in a topologically disordered system

Note added in proof My attention has been diawn to interesting eallier work by Fiadkov, Shvindlerman, and Udler $^{38}$ on this grain giowth model These authors stud1ed single-grain properties, but did not consider correlations between adjacent grains
${ }^{1}$ See the review article by $\mathrm{D}$ Wedre and $\mathrm{N}$ Rivier, Contemp Phys 25, 59 (1984)

${ }^{2}$ D A Aboav, Metallography 3, 383 (1970)

${ }^{3} \mathrm{D}$ Wearre, Metallogt aphy 7, 157 (1974)

${ }^{4} \mathrm{~N}$ Riviet, Philos Mag B 52, 795 (1985)

${ }^{5} \mathrm{R}$ Kikuch, J Chem Phys 24, 861 (1956)

${ }^{6} \mathrm{P}$ Feltham, Acta Metall 5, 97 (1957)

${ }^{7} \mathrm{M}$ Hillert, Acta Metall 13, 227 (1965)

${ }^{8} \mathrm{~N}$ P Louat, Acta Metall 22, 721 (1974)

${ }^{9} \mathrm{~F}$ N Rhines and K R Craig, Metall Trans 5, 413 (1974)

${ }^{10} \mathrm{P}$ S Sahni, G S Grest, M P Anderson, and D J Srolovitz, Phys Rev Lett 50, 263 (1983), Acta Metall 32, 793 (1984), 32, 793 (1984), P S Sahn1, D J Srolovitz, G S Grcst, M P Anderson, and S A Safran, Phys Rev B 28, 2705 (i983)

$11 \mathrm{~J}$ Wejchert, D Wcarre, and J P Keımode, Philos Mag B 53, 15 (1986)

${ }^{12}$ W W Mullins, J Appl Phys 27, 900 (1956)

${ }^{13} \mathrm{~J}$ von Neumann, in Metal Interfaces, edited by C Heiring (American Society for Metals, Cleveland, 1952), p 108

${ }^{14}$ M Marder, Phys Rev A 36, 438 (1987)

${ }^{15}$ C W J Beenakker, Phys Rev A 33, 4482 (1986)

16Y Enomoto, M Tokuyama, and K Kawasak1, Acta Metall 34, 2119 (1986)

${ }^{17}$ M Maider, Phys Rev A 36, 858 (1987)

${ }^{18} \mathrm{D}$ Wcalse and J P Kermode, Philos Mag B 47, L29 (1983)

${ }^{19} \mathrm{H}$ Furukawa, Adv Phys 34, 703 (1985)

${ }^{90} \mathrm{C}$ W I Beenakker, Phys Rev Lett 57, 2454 (1986)

$21 \mathrm{~J}$ A Glazier, S P Gioss, and J Stavans, Phys Rev A 36, 306 (1987)

22I W Chen, Acta Metall 35, 1723 (1987)
${ }^{24}$ V Yu Novikov, Acta Metall 26, 1739 (1978), 27, 1461 (1979), O Hunderı and N Ryum, Acta Metall 27, 161 (1979), 29, 1737 (1981)

${ }^{25}$ E A Ceppi and O B Nasello, Scr Metall 18, 1221 (1984), A Soares, A C Ferro, and M A Fortes, tbıd 19, 1491 (1985), H J Frost, C V Thompson, C L Howe, and J Whang (unpublished)

${ }^{26}$ D Weaure and J P Kermode, Phılos Mag B 48, 245 (1983), 50, 379 (1984)

${ }^{27} \mathrm{M}$ P Anderson, G S Giest, and D J Srolovitz, Scr Metall 19, 225 (1985)

${ }^{28}$ The initial state is obtained by peiforming a given number of $\mathrm{T} 1$ processes at 1 andom on grains in a hexagonal network In the ordered case $(O)$, the simulation then starts with $90 \%$ of hexagonal grams, and with an arca distribution which has a standard deviation of $10 \%$ of its average In the disordered case $(D)$, these values are, respectively, $40 \%$ and $60 \%$

${ }^{29}$ Occasionally, some grain $l$ has two sides in common with the same neighbor I then follow the convention of counting that neighbor twice when calculating $\rho$ and $v$

${ }^{30} \mathrm{C} \mathbf{J}$ Simpson, C J Beingessner, and W C Winegard, AIME Trans 239, 587 (1967)

${ }^{31}$ C W J Beenakker, Physica A 147, 256 (1987)

${ }^{32} \mathrm{D}$ A Aboav, Metallography 13, 43 (1980)

${ }^{33} \mathrm{C}$ J Lambert and D Weare, Metallography 14, 307 (1981), Philos Mag B 47, 445 (1983)

${ }^{34}$ In Ostwald ripening, the size correlation coefficient is about -02 for near by dioplets, at a precipitate volume fiaction of $10 \%$ (see Ref 15 )

${ }^{35}$ This has been shown by explicit calculation by W W Mullins, 
J. Appl. Phys. 59, 1341 (1986).

${ }^{36}$ G. S. Grest, M. P. Anderson, and D. J. Srolovitz, in Proceedings of the NATO Conference on Time-Dependent Effects in Disordered Materials, Geilo, Norway, 1987 (to be published).
${ }^{37}$ Recrystallization of Metallic Materials, edited by F. Haessner (Riederer, Stuttgart, 1978).

${ }^{38}$ V. E. Fradkov, L. S. Shvindlerman, and D. G. Udler, Scr. Metall. 19, 1285 (1985). 